Check for updates

Cite this: RSC Adv., 2019, 9, 15392

\title{
Metabolism and pharmacokinetics of medium chain fatty acids after oral administration of royal jelly to healthy subjects
}

\author{
Masayuki Yamaga, (D) * Hiroko Tani, Ayanori Yamaki, Tomoki Tatefuji \\ and Ken Hashimoto
}

\begin{abstract}
The unique fatty acids in royal jelly (RJ), 10-hydroxy-2-decenoic acid and 10-hydroxydecanoic acid are expected to be associated with many health benefits, but little is known on the pharmacokinetics and metabolism. The aim of this study is to confirm the metabolism and pharmacokinetics of RJ fatty acids in humans. Twelve volunteers received RJ capsules or enzyme treated RJ (ETRJ) capsules (800 mg). The other group received two doses of ETRJ tablets $(800 \mathrm{mg}$ and $1600 \mathrm{mg}$ ). Plasma samples were collected up to $12 \mathrm{~h}$ after the RJ intake and urine samples were collected within $24 \mathrm{~h}$ after ETRJ tablet consumption. The samples were analyzed by LC/MS/MS. A multivariate analysis of the RJ dose plasma samples detected 2-decenedioic acid (2-DA), sebacic acid (SA), and 3-hydroxysebacic acid (3-HSA) with significantly different intensities $(P<0.05)$ before and after RJ intake. The area under the concentration (AUC) of 2-DA, SA, and 3-HSA was $2500.05 \pm 569.58,322.57 \pm 137.36$, and $242.98 \pm 58.36 \mathrm{ng} \mathrm{h} \mathrm{mL}^{-1}$, respectively. By enzyme treatment, the AUC of 2-DA, SA, and 3-HSA was significantly increased $(P<$ 0.05). The values of AUC and urinary excretion of these metabolites were dose-dependent. The major RJ fatty acids were metabolized to dicarboxylate, absorbed into the circulation and their absorption increased by enzyme treatment. This study provides useful information that will support studies aimed at clarifying the identity of bioactive RJ constituents and their biological effect, and further the development of RJ.
\end{abstract}

Received 22nd April 2019 Accepted 10th May 2019 DOI: 10.1039/c9ra02991e rsc.li/rsc-advances

\section{Introduction}

Royal jelly (RJ) is a secretion product of the cephalic glands of honey bees (Apis mellifera $\mathrm{L}$.) and is one of the most attractive functional foods. It is the specific food for the queen honey bee throughout her life period and it is involved in the fertility and longevity of the queen. ${ }^{1}$

A multitude of pharmacological activities have been reported in experimental animals ${ }^{2-6}$ and in clinical trials, ${ }^{7-10}$ including antitumor, ${ }^{2}$ anti-oxidant, ${ }^{3}$ anti-inflammatory, ${ }^{4}$ antibacterial, ${ }^{5}$ and anti-aging, ${ }^{6}$ improvement of chills, ${ }^{7}$ neck muscle strain, ${ }^{8}$ tinnitus, ${ }^{9}$ female menopausal symptoms, ${ }^{10}$ age associated muscle strength decline, ${ }^{11}$ and lipoprotein metabolism by lowering serum total cholesterol and leading to low-density lipoprotein levels, as well. ${ }^{12}$

The chemical composition of RJ consists of: water (60$70 \%)$, proteins (9-18\%), sugars $(7.5-23 \%)$, lipids (3-8\%), and other trace compounds. The two major unique fatty acids in $\mathrm{RJ}$ are trans-10-hydroxy-2-decenoic acid (10H2DA) and 10hydroxydecanoic acid (10HDAA) which comprise $60-80 \%$ of

Institute for Bee Products and Health Science, Yamada Bee Company, Inc., 194 Ichiba, Kagamino-cho, Tomata-gun, Okayama, Japan.E-mail: my1636@yamada-bee.com; Tel: $+81-868-54-1199$
RJ lipids. ${ }^{13}$ Moreover, the characterizations of fatty acids composition in RJ have been determined as a set of $\mathrm{C} 8, \mathrm{C} 10$, and C12 fatty acids. ${ }^{14}$ Several pharmacological activities of 10H2DA and 10HDAA have been reported in animal experiments ${ }^{15-19}$ and these fatty acids are expected to play an important role in the pharmacological activities of RJ.

Generally, medium chain fatty acids (MCFAs) containing 8-12 carbons are absorbed more efficiently than long chain fatty acids and transported in the portal blood directly to the liver, unlike long chain fatty acids. Subsequently, MCFAs enter the mitochondria independently of the carnitine transport system and undergo preferential $\beta$-oxidation; the resulting glut of acetyl-CoA derives ketone body production, being linked to many health benefits. ${ }^{20}$ However, there is little known on the pharmacokinetics of the MCFAs from RJ. In addition, the pharmacokinetic influence of protease treated RJ, which has been reported to reduce the immunoreactivity and allergenicity of $\mathrm{RJ}^{21}$ has not been investigated yet. The aim of this study is to confirm the metabolism and pharmacokinetics of RJ fatty acids in humans. The effect of protease treatment of RJ on the fatty acids absorption is also investigated. 
Table 1 Clinical and demographic characteristics of study participants $^{a}$

\begin{tabular}{llllll}
\hline Group & $n$ & Age & Height $(\mathrm{cm})$ & Weight $(\mathrm{kg})$ & BMI $\left(\mathrm{kg} \mathrm{m}^{-2}\right)$ \\
\hline A (Female) & 6 & $27.8 \pm 1.3$ & $157.2 \pm 3.1$ & $55.3 \pm 1.2$ & $22.4 \pm 1.0$ \\
A (Male) & 6 & $27.5 \pm 2.1$ & $174.0 \pm 4.3$ & $68.2 \pm 7.0$ & $22.5 \pm 2.1$ \\
B (Female) & 6 & $27.0 \pm 0.6$ & $156.8 \pm 2.9$ & $53.7 \pm 4.1$ & $21.9 \pm 1.9$ \\
B (Male) & 6 & $27.2 \pm 2.2$ & $173.4 \pm 4.1$ & $66.2 \pm 5.3$ & $22.0 \pm 1.7$
\end{tabular}

${ }^{a}$ All values are mean \pm SDs; Group A, the subjects received RJ and ETRJ capsules; Group B, the subjects received two dose of ETRJ (800 mg and $1600 \mathrm{mg}$ ); BMI, body mass index; ETRJ, enzyme treated royal jelly; RJ, royal jelly.

\section{Materials and methods}

\subsection{Chemicals and materials}

LC/MS grade methanol was obtained from Kanto Chemical Co., Inc. (Tokyo, Japan) and LC/MS grade acetonitrile and HPLC grade acetic acid were obtained from Wako Pure Chemical Industries, Ltd. (Osaka, Japan). 10H2DA was purchased from Hangzhou Eastbiopharm, Co.,Ltd. (Hangzhou, China), and 10HDAA was purchased from Combi-Blocks, Inc. (San Diego, USA). 2-Decenedioic acid (2-DA) was purchased from Sundia MediTech Co., Ltd. (Shanghai, China); decanedioic acid (sebacic acid, SA) and 8-hydroxyoctanoic acid were purchased from Sigma-Aldrich Co., LLC. (St. Louis, USA); 3-hydroxysebacic acid (3-HSA) was purchased from Santa Cruz Biotechnology (Dallas, USA); 12-hydroxydodecanoic acid was purchased from MP Biomedicals, LLC. (California, USA); traumatic acid was purchased from Cayman Chemical (Ann Arbor, USA). Dodecenedioic acid and trans-2-heptenoic acid were purchased from Tokyo Chemical Industry Co., Ltd. (Tokyo, Japan). 3,10-Dihydroxydecanoic acid was isolated from RJ and obtained by HRFABMS and ${ }^{13} \mathrm{C}$ NMR data. The spectroscopic data were completely identical to those reported by Melliou and

A
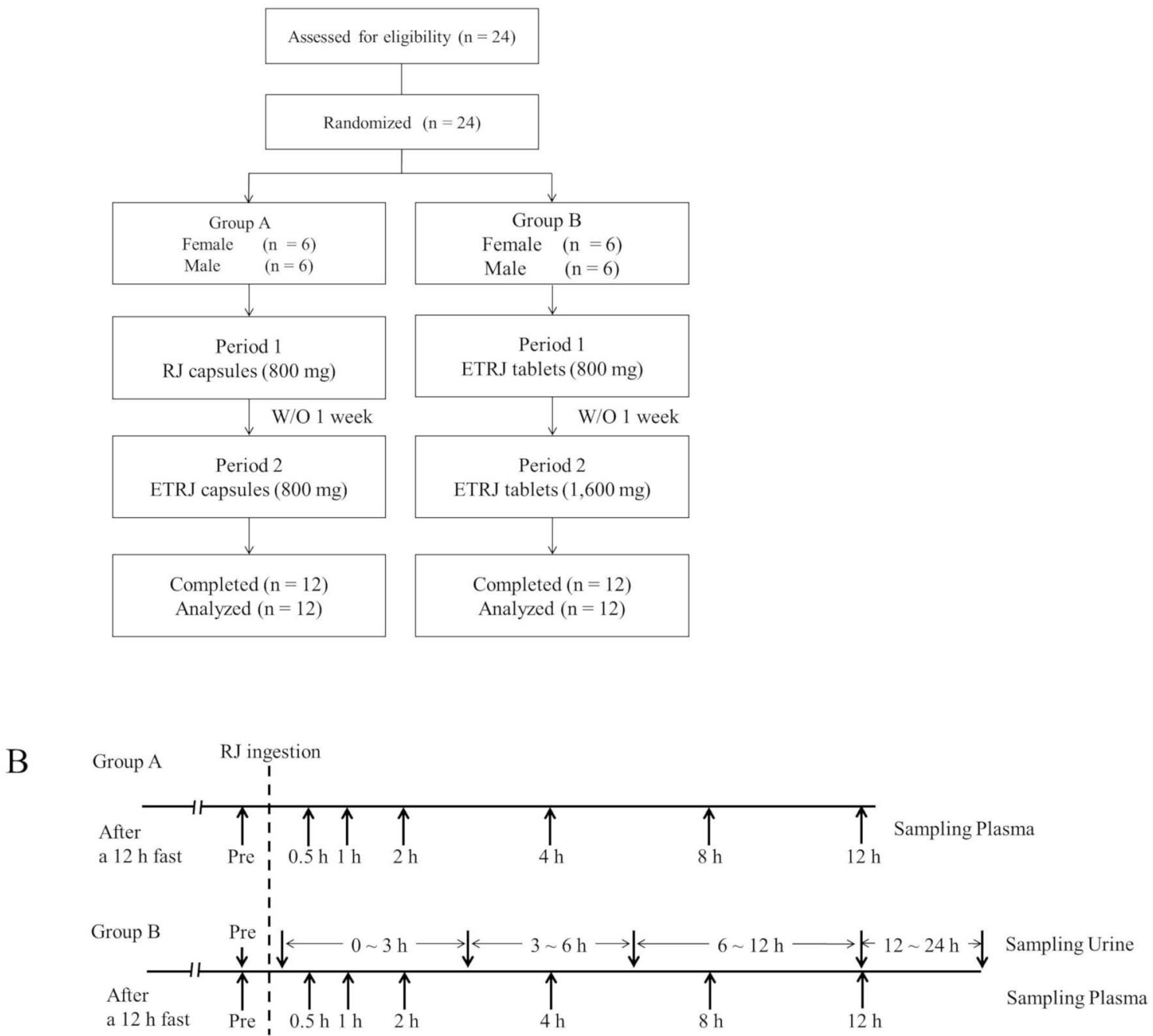

Fig. 1 (A) Flow diagram of participants through the study. ETRJ, enzyme treated royal jelly; RJ, royal jelly; W/O, wash out. (B) Schematic representation of the study design. The subjects ingested RJ for at least $12 \mathrm{~h}$ after fasting. The blood samples were obtained before and 30 min, $1 \mathrm{~h}, 2 \mathrm{~h}, 4 \mathrm{~h}, 8 \mathrm{~h}$, and $12 \mathrm{~h}$ after RJ intake. In group B, the urine samples were collected before RJ intake and between $0-3,3-6,6-12$, and $12-24 \mathrm{~h}$ after RJ intake. 
Table 2 Content of fatty acids in RJ used in clinical trials ${ }^{a}$

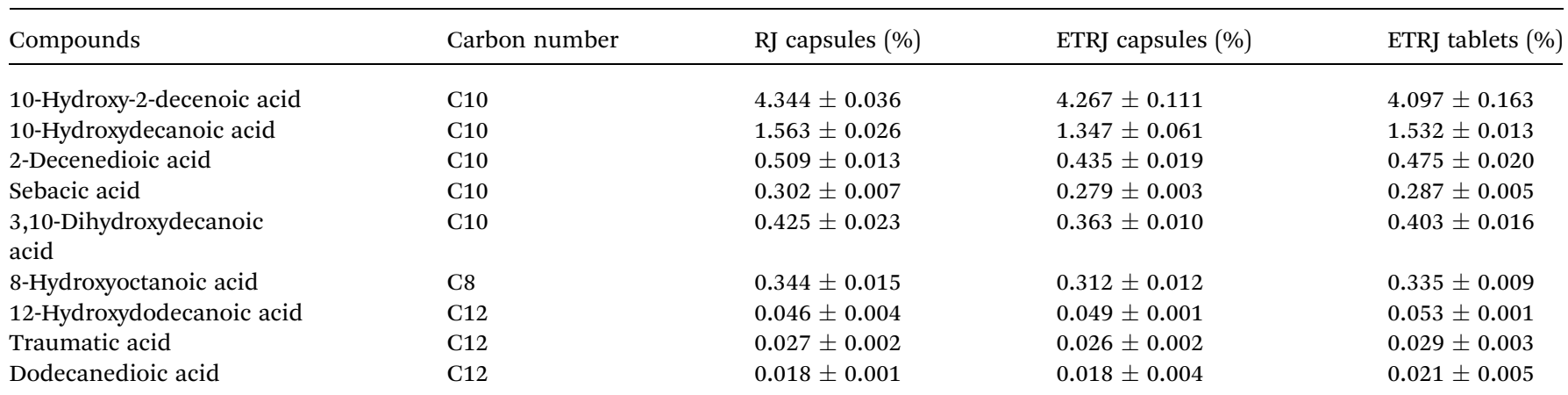

${ }^{a}$ All values are means $\pm \mathrm{SDs} ; n=3$. ETRJ, enzyme treated royal jelly. RJ, royal jelly.

Chunou. ${ }^{22}$ The RJ powder was obtained by freeze drying of RJ and the enzyme treated RJ (ETRJ) powder was obtained by degradation of RJ protein using protease and freeze drying. The RJ powder standardized to contain minimum 3.8\% 10H2DA, minimum $0.6 \%$ 10HDAA and the ETRJ powder standardized to contain minimum 3.5\% 10H2DA, minimum 0.6\% 10HDAA, respectively. The capsules were prepared by encapsulating $\mathrm{RJ}$ or ETRJ powder in hard capsules and ETRJ tablets were sugar coated containing $9.2 \%$ diluents. Both capsules and tablets were obtained from Yamada Bee Company, Inc.

\subsection{Extraction of RJ fatty acids}

Each RJ sample (200 mg) used in clinical trials was extracted with $3 \mathrm{~mL}$ methanol and centrifuged at $2000 \times g$ for $10 \mathrm{~min}$ at room temperature; the supernatant was collected into a new vial. The same extraction was repeated three times and collected supernatant was diluted with methanol up to a concentration of $10 \mu \mathrm{g} \mathrm{mL} \mathrm{m}^{-1}$. The extracts were filtered with a $0.2 \mu \mathrm{m}$ filter (DISMIC 13HP, Advantec, Tokyo, Japan), and $3 \mu \mathrm{L}$ of the filtrates were injected into LC/MS/MS system.

\subsection{Study design and sampling}

Twelve volunteers (Group A, 6 men and 6 women, aged 26-33 y) with no apparent diseases participated in this study. One week before the study and during the study, the volunteers were directed not to take RJ, supplements or drinks including RJ. Baseline samples were collected after a $12 \mathrm{~h}$ evening fast. The subjects received RJ capsules or ETRJ capsules (800 mg - 4 capsules) in 2 experimental periods separated by 6 day wash-out period. Further, the other twelve volunteers (Group B, 6 men and 6 women, aged 26-32 y) received two doses of ETRJ tablets (800 mg - 3 tablets and $1600 \mathrm{mg}-6$ tablets) in 2 experimental periods separated by 6 day wash-out period. Table 1 shows demographics of the subjects in each group. Blood samples were collected before the RJ intake and at $30 \mathrm{~min}, 1,2,4,8$, and $12 \mathrm{~h}$ after the RJ intake (Fig. 1). The collected blood samples were centrifuged at $2000 \times g$ for $3 \mathrm{~min}$ at room temperature. The urine samples were obtained before RJ consumption and between $0-3,3-6,6-12$, and 12-24 $\mathrm{h}$ after the ETRJ tablets consumption. The obtained plasma and urine samples were stored at $-80{ }^{\circ} \mathrm{C}$ until LC/MS/MS analysis. This study design was carried out in accordance with the principles outlined in the Declaration of Helsinki and approved by Yamada Bee Co. Ethics Committee (Registered Japan Agency for Medical Research and Development, No. 16000050). All of the subjects gave their written informed consent.

\subsection{Plasma and urine samples preparation}

For LC/MS/MS analysis, $50 \mu \mathrm{L}$ of plasma were mixed with $500 \mu \mathrm{L}$ methanol and centrifuged at $10000 \times g$ for $5 \mathrm{~min}$ at room temperature. The collected supernatant was evaporated with nitrogen gas and dissolved with $150 \mu \mathrm{L}$ methanol. The samples were filtered with a $0.2 \mu \mathrm{m}$ filter, and $3 \mu \mathrm{L}$ of the filtrates were injected into LC/MS/MS system. Two hundreds $\mu \mathrm{L}$ of urine were centrifuged at $10000 \times g$ for $5 \mathrm{~min}$ at room temperature and the supernatant was diluted with $800 \mu \mathrm{L}$ methanol. Centrifugation was performed under the same conditions; the supernatants were evaporated with nitrogen gas and dissolved with $200 \mu \mathrm{L}$ of $50 \%$ methanol solution. The samples were filtered and $3 \mu \mathrm{L}$ were injected into LC/MS/MS system.

\subsection{LC/MS/MS analysis}

LC analysis was performed using an Ultimate3000 system (Thermo Scientific). Separation of metabolites was performed at $40{ }^{\circ} \mathrm{C}$ using a reversed phase column (Acquity UPLC BEH C18, $2.1 \times 100 \mathrm{~mm}, 1.7 \mu \mathrm{m}$ i.d. Waters). Injections were carried out with an autosampler maintained at $5{ }^{\circ} \mathrm{C}$. The mobile phase consisting of water with $0.01 \%$ acetic acid (A) and acetonitrile (B) was pumped at a flow rate of 0.3 $\mathrm{mL} \min ^{-1}$. The gradient system was as follows: $5 \% \mathrm{~B}(0-2$ $\min$ ), 5-100\% B (2-22 min), 100\% B (22-25 min), 5\% B (25-30 min). MS/MS was performed on a Q-Exactive focus Orbitrap type mass spectrometer (Thermo Scientific) equipped with heated electrospray ion source (HESI) in the positive and negative modes. Mass detection and quantification of analytes were accomplished in full scan (70 000 resolution FWHM at $m / z 200$, mass range of $m / z 70-1000)$ and data dependent MSMS (13 500 resolution FWHM at $m / z 200$ ). Ion source conditions for both ionization polarities were: spray voltage $3.5 \mathrm{kv}$ (positive), $2.5 \mathrm{kv}$ (negative), sheath gas 50 
I

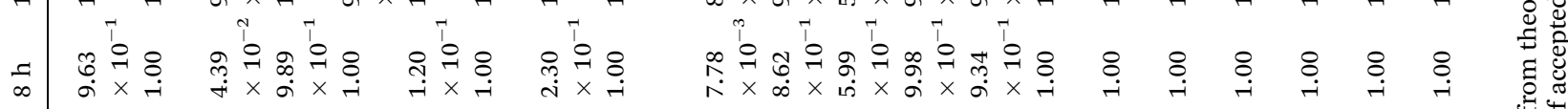

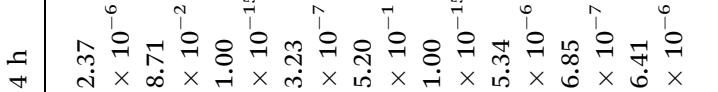

\&ํํำ

章

$x-x$

$\overbrace{0}^{\circ} \frac{10}{n}$

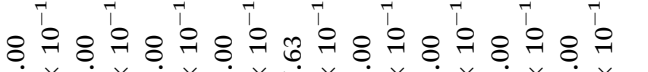

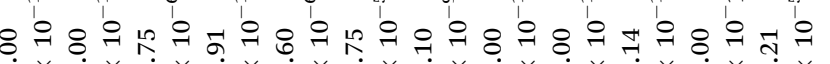

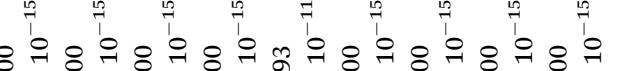

일

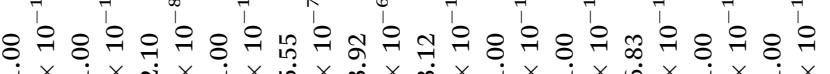

1
10

की

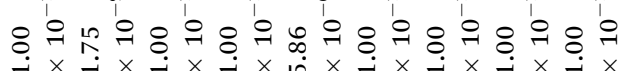

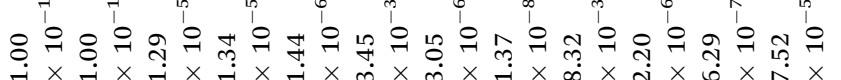

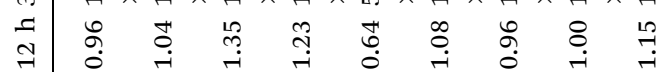

$$
\text { 芒 }
$$

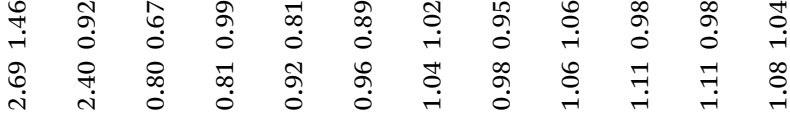

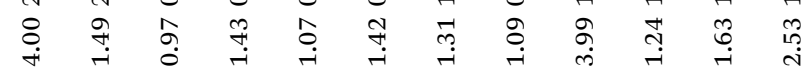$$
7
$$$$
\stackrel{0}{1} \stackrel{0}{1}
$$

$\stackrel{+}{\circ}$

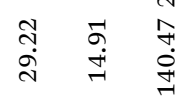

齐

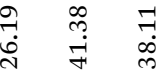

r

$+e^{+}$

ำ

No

i

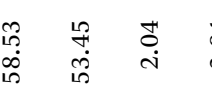

अా

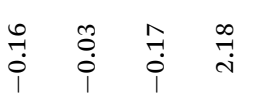

로

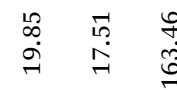

ळे

$\begin{array}{ll}0 & 0 \\ \dot{m} & \stackrel{-}{-}\end{array}$



के

$\stackrel{\infty}{1}$

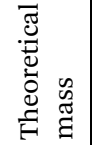

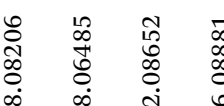

雚

帘

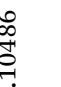

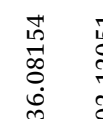

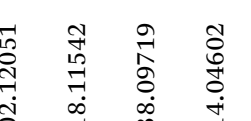

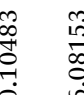

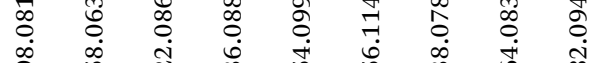

宛

คे

宁空

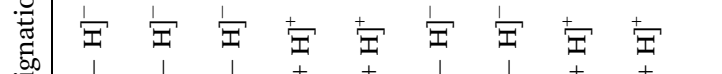

I

ลे

苟

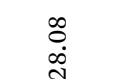

(1)

in

兽

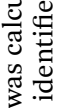

造.

离蓠

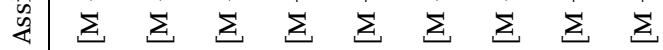

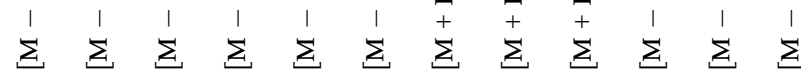

医

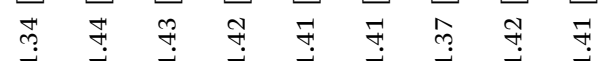

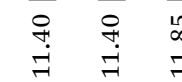
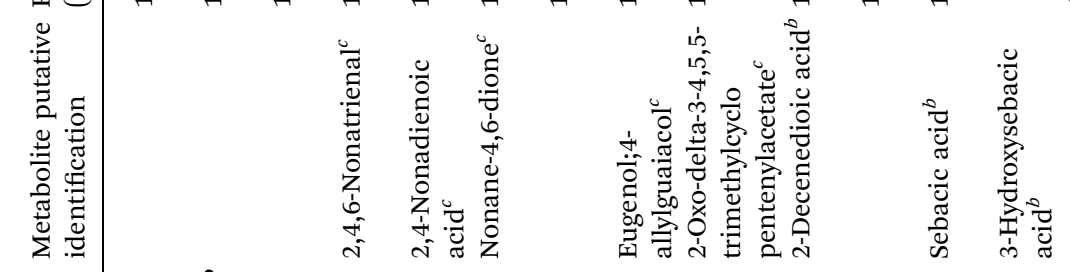

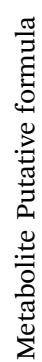

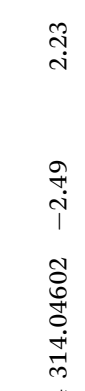

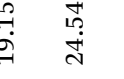

苛

嵌

离

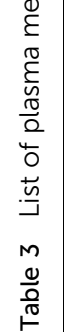
$0^{2} e^{\infty}$

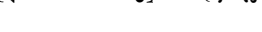
$\begin{array}{lll}0_{1} & 0^{\infty} & \\ 0^{\infty} & z^{-1} & 0^{N} \\ z^{\infty} & 0 & z^{\infty}\end{array}$
I

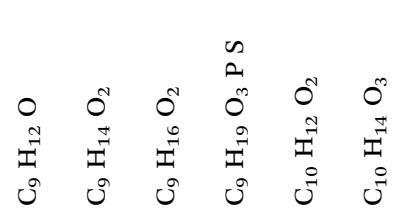
$\begin{array}{llll}\text { का } & 0 & & \\ 0 & 0 & 0 & 0 \\ 0 & 0 & 0 \\ 0 & z^{\infty} & z^{10} & z^{\circ}\end{array}$

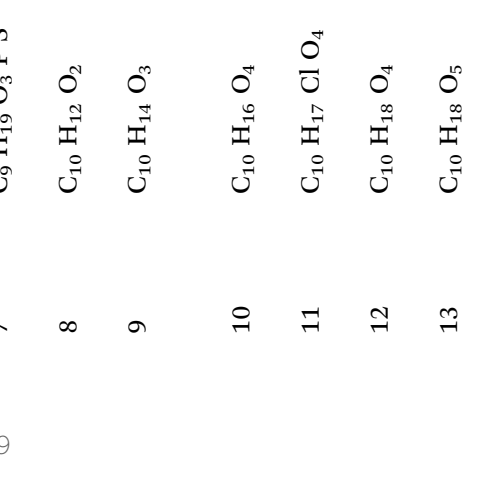

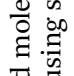

ซึ.

듐

8

苞

荧离 

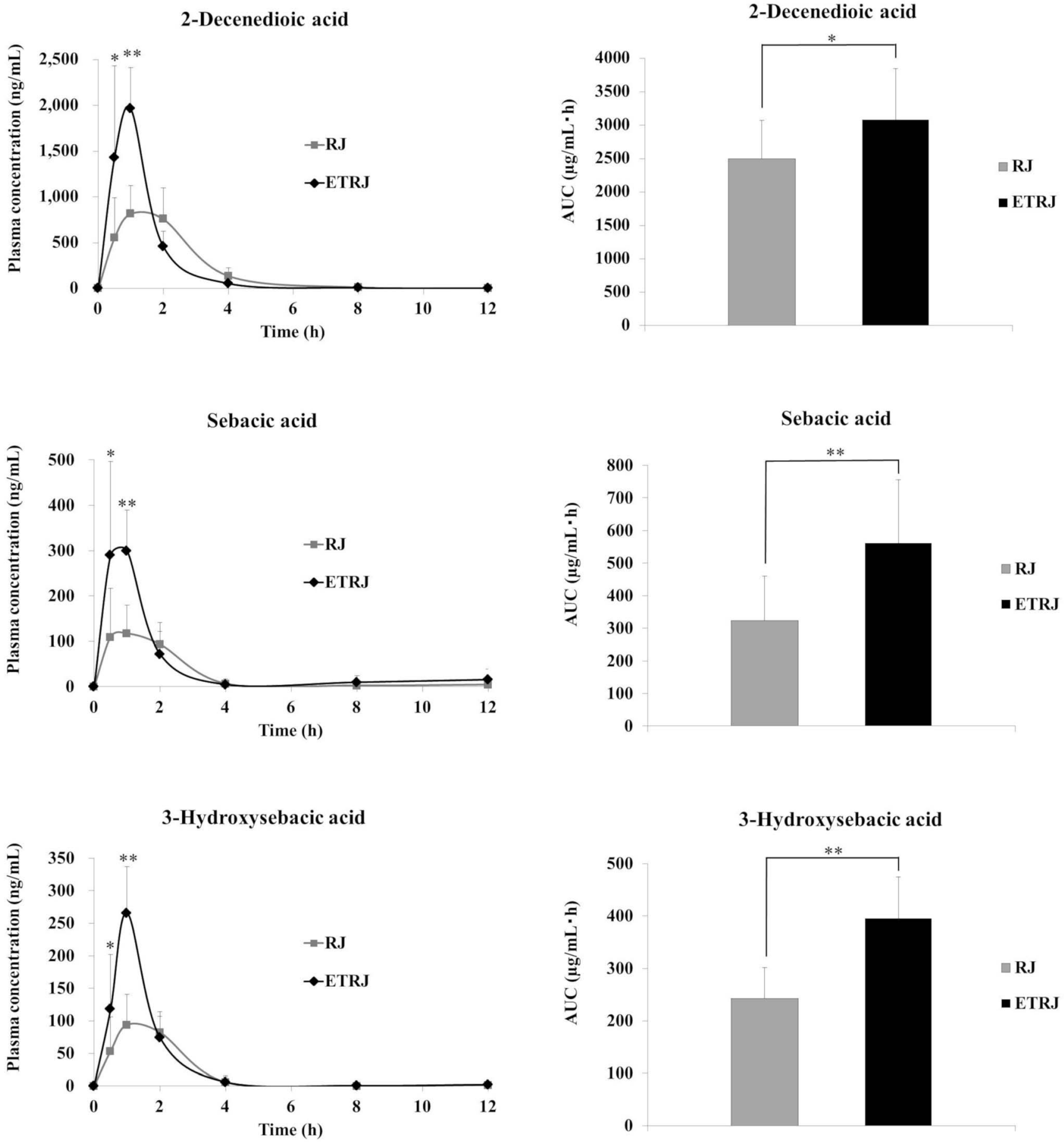

Fig. 2 Plasma concentration-time curve and AUC of 2-decenedioic acid, sebacic acid, and 3-hydroxysebacic acid in human subjects (mean \pm

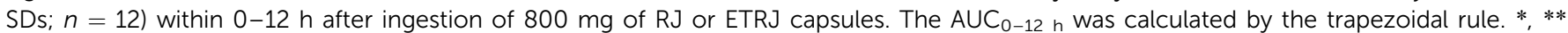
Significantly different between RJ and ETRJ, *: $P<0.05$ and **: $P<0.01$ (paired $t$ test).

arbitrary units, auxillary gas: 10 arbitrary units, sweep gas: 0 arbitrary units, heater temperature: $400{ }^{\circ} \mathrm{C}$, capillary temperature: $250^{\circ} \mathrm{C}$, S-lens RF level: 50 , and collision energy: $15,30,45 \mathrm{eV}$ (normalized stepped collision). The instrument was calibrated using Ultramark 1621 (Thermo Fisher Scientific) prior to the beginning of the analysis.

\subsection{Data processing and analysis}

Thermo Scientific Compound Discoverer version 2.1 software was used for comparative analysis. Results from differential analysis of aligned chromatographic peaks were filtered requiring a minimum 2-fold change in peak area and significant increase in peak area between RJ capsules pre-dose and after-dose samples 
Table 4 Pharmacokinetic parameters of plasma fatty acid metabolites of human volunteers after consumption of 800 mg RJ and ETRJ capsules ${ }^{a}$

\begin{tabular}{|c|c|c|c|c|c|c|c|c|}
\hline Metabolites & \multicolumn{4}{|l|}{ RJ $800 \mathrm{mg}$} & \multicolumn{4}{|c|}{ ETRJ $800 \mathrm{mg}$} \\
\hline Sebacic acid & $1.33 \pm 0.59$ & 1.26 & $117.14 \pm 61.73$ & $322.57 \pm 137.36$ & $0.83 \pm 0.24$ & 1.39 & $299.86 \pm 89.81$ & $561.08 \pm 193.83$ \\
\hline 3-Hydroxysebacic acid & $1.38 \pm 0.54$ & 1.04 & $94.13 \pm 46.87$ & $242.98 \pm 58.36$ & $0.92 \pm 0.19$ & 0.93 & $265.78 \pm 71.26$ & $395.77 \pm 78.93$ \\
\hline
\end{tabular}

${ }^{a}$ All values are means $\pm \operatorname{SDs}(n=12)$. $C_{\max }$, maximum plasma concentration; $T_{\max }$, time to reach $C_{\max }$, AUC, area under the curve $(0-12 \mathrm{~h}) ; t_{1 / 2}$, elimination half-life.

(student's $t$ test $P<0.05$ ). The metabolites have been presumed on the basis of their exact mass, which was compared to those registered in the Chemspider (http://www.chemspider.com), the Kyoto Encyclopedia of Genes and Genomes (http:// www.genome.jp), Human Metabolome Database (http:// www.hmdb.ca/) and mzCloud (https://www.mzcloud.org). Quantification of fatty acids (10H2DA, 10HDAA, 2-DA, SA, and 3-HSA) was performed using the plasma and/or urine matrix standard curves. The standard curves were obtained by analyzing standard samples with concentrations ranging from 5 to $2000 \mathrm{ng} \mathrm{mL}{ }^{-1}$. Pharmacokinetic analysis, maximum plasma concentration $\left(C_{\max }\right)$ and the time to reach $C_{\max }\left(T_{\max }\right)$ were taken directly from observed plasma concentration-time profiles. The elimination half-life $\left(t_{1 / 2}\right)$ for the RJ fatty acids was calculated as $0.693 / K_{\mathrm{el}}$, where $K_{\mathrm{el}}$ is the slope of the linear regression of the plasma concentration. The area under the plasma concentration-time curve ( $\mathrm{AUC}_{0-12 \mathrm{~h}}, \mathrm{ng} \mathrm{h} \mathrm{mL} \mathrm{m}^{-1}$ ) was calculated by the trapezoidal rule up to the last measured data point, $12 \mathrm{~h}$. Data on plasma concentration and AUC values of 2-DA, SA, and 3-HSA acid after ingestion of RJ capsules and ETRJ capsules were subjected to statistical analysis by using analysis of variance and a paired $t$ test with JMP for Windows 5 (SAS Institute Japan, Tokyo, Japan).

\section{Results}

\subsection{Fatty acids content in RJ}

As shown in Table 2, the fatty acids content in RJ capsules, ETRJ capsules, and ETRJ tablets were as follows: 4.1-4.3\% 10H2DA $\left(\mathrm{m} / \mathrm{z}: 185.1183[\mathrm{M}-\mathrm{H}]^{-}\right), 1.3-1.6 \%$ 10HDAA $(\mathrm{m} / \mathrm{z}: 187.1340$ $\left.[\mathrm{M}-\mathrm{H}]^{-}\right), 0.44-0.51 \%$ 2-DA $\left(m / z: 199.0976[\mathrm{M}-\mathrm{H}]^{-}\right), 0.28-0.3 \%$ SA ( $\left.m / z: 201.1132[\mathrm{M}-\mathrm{H}]^{-}\right), 0.36-0.43 \%$ 3,10-dihydroxydecanoic acid $\left(m / z: 203.1289[\mathrm{M}-\mathrm{H}]^{-}\right), 0.31-0.34 \%$ 8-hydroxyoctanoic acid $\left(\mathrm{m} / \mathrm{z}: 159.1027[\mathrm{M}-\mathrm{H}]^{-}\right), 0.046-0.053 \%$ 12-hydroxydodecanoic acid, $0.026-0.029 \%$ traumatic acid (2-dodecenedioic acid, $\left.m / z: 227.1289[\mathrm{M}-\mathrm{H}]^{-}\right)$, and $0.018-0.021 \%$ dodecanedioic acid $\left(m / z: 229.1445[\mathrm{M}-\mathrm{H}]^{-}\right)$, respectively.

\subsection{Qualitative analysis of $\mathrm{RJ}$ metabolites in plasma}

The blood samples were collected up to $12 \mathrm{~h}$ from subjects who received $800 \mathrm{mg}$ RJ capsules. The RJ metabolites have been analyzed by LC/MS/MS. A multivariate analysis of the plasma samples did not reveal the two major fatty acids, 10H2DA and 10HDAA, but revealed the detection in positive and/or negative modes of 21 metabolites with significantly different intensities ( $P$ value $<0.05$ and 2 hold change) before and after RJ capsules intake (Table 3). Nine of the 21 metabolites have been putatively identified on the basis of their exact mass, which was compared to those registered in online databases. Three of the putatively identified metabolites were indicated by LC retention time and MSMS fragmentation pattern of standards; 2-DA, SA, and 3-hydroxy derivative of SA $\left(m / z: 217.1082[\mathrm{M}-\mathrm{H}]^{-}\right)$.

\subsection{Pharmacokinetics of RJ fatty acid metabolites}

The plasma concentration-time profiles of identified fatty acid metabolites in plasma after the intake of RJ and ETRJ capsules are presented in Fig. 2 and Table 4. The $C_{\max }$ value of 2-DA, SA, and 3-HSA was $815.23 \pm 311.13 \mathrm{ng} \mathrm{mL}^{-1}, 117.14 \pm 61.73 \mathrm{ng}$ $\mathrm{mL}^{-1}$, and $94.13 \pm 46.87 \mathrm{ng} \mathrm{mL}^{-1} 1 \mathrm{~h}$ after ingestion of $800 \mathrm{mg}$ RJ capsules, respectively. The AUC $0-12 \mathrm{~h}$ of 2-DA, SA, and 3-HSA was $2500.05 \pm 569.58 \mathrm{ng} \mathrm{h} \mathrm{mL}^{-1}, 322.57 \pm 137.36 \mathrm{ng} \mathrm{h} \mathrm{mL}^{-1}$, and $242.98 \pm 58.36 \mathrm{ng} \mathrm{h} \mathrm{mL}{ }^{-1}$, respectively. Meanwhile, the plasma concentration of 2-DA at $30 \mathrm{~min}$ and $1 \mathrm{~h}$ after ingestion of ETRJ capsules was significantly higher than after the RJ capsules intake (paired $t$ test $P<0.05, P<0.01$ ). The $\mathrm{AUC}_{0-12 \mathrm{~h}}$ of 2-DA indicated a significant difference after the ETRJ capsules intake as compared to RJ capsules (paired $t$ test $P<0.05$ ). The plasma concentration of SA and 3-HSA at $30 \mathrm{~min}$ and $1 \mathrm{~h}$ after ingestion of ETRJ capsules was significantly higher than after the RJ capsules intake (paired $t$ test $P<0.05, P<0.01$ ). The AUC values of SA and 3-HSA after the ETRJ capsules intake were significantly different compared with those obtained after the RJ capsules intake (paired $t$ test $P<0.01$ ).

\subsection{Dose dependency of plasma concentration and urine excretion of RJ fatty acids}

Fig. 3 and 4 show the plasma concentration and urinary excretion of RJ fatty acid metabolites after ingestion of two doses of ETRJ tablets. The $C_{\max }$ of 2-DA, SA, and 3-HSA after ingestion of $1600 \mathrm{mg}$ ETRJ tablets was $2482.99 \pm 1083.75 \mathrm{ng}$ $\mathrm{mL}^{-1}, 484.80 \pm 211.47 \mathrm{ng} \mathrm{mL}{ }^{-1}$, and $258.74 \pm 147.06 \mathrm{ng}$ $\mathrm{mL}^{-1}$, respectively. The AUC ${ }_{0-12 \mathrm{~h}}$ of these metabolites after ingestion of $1600 \mathrm{mg}$ ETRJ tablets was $5444.44 \pm 1614.27 \mathrm{ng} \mathrm{h}$ $\mathrm{mL}^{-1}, 1026.47 \pm 409.31 \mathrm{ng} \mathrm{h} \mathrm{mL}^{-1}$, and $642.01 \pm 218.50 \mathrm{ng} \mathrm{h}$ $\mathrm{mL}^{-1}$, respectively. There were no significant difference in $C_{\max }$ and AUC value of RJ fatty acid metabolites between men and women (Table 5, student's $t$ test $P>0.1$ ). Further, urinary metabolites of five urine fractions collected within $24 \mathrm{~h}$ after 

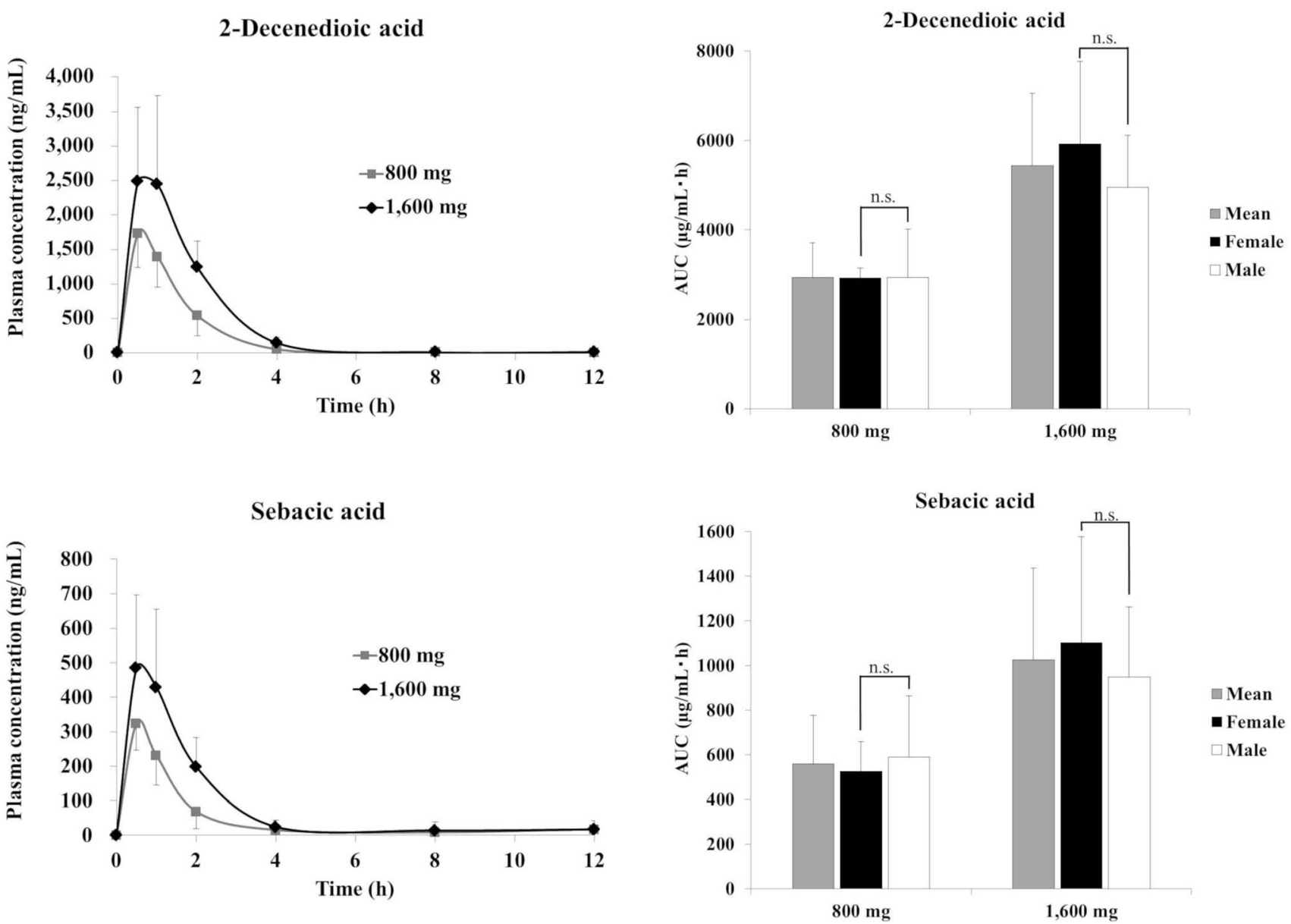

3-Hydroxysebacic acid
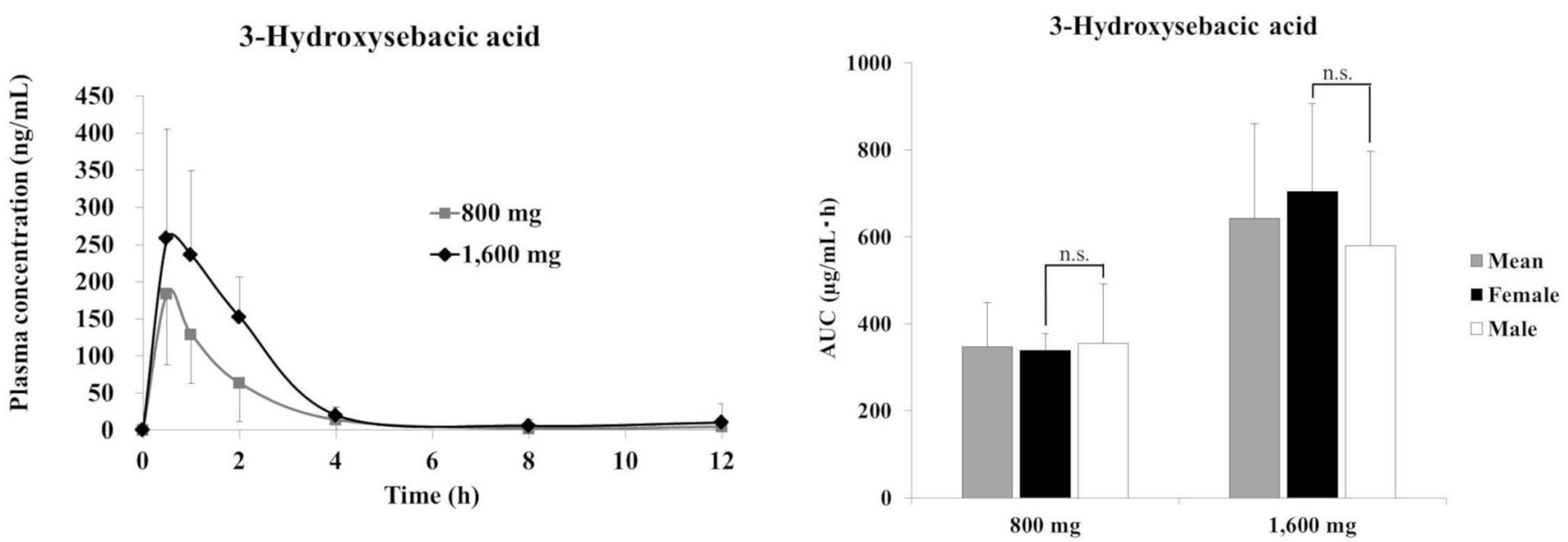

Fig. 3 Plasma concentration time curve and AUC of 2-decenedioic acid, sebacic acid, and 3-hydroxysebacic acid in human subjects (mean \pm SDs; $n=12$ ) within $0-12 \mathrm{~h}$ after ingestion of $800 \mathrm{mg}$ or $1600 \mathrm{mg}$ ETRJ tablets. The $\mathrm{AUC}_{0-12 \mathrm{~h}}$ was calculated by the trapezoidal rule. $\mathrm{n} . \mathrm{s} ., \mathrm{not}$ significant (student's $t$ test).

the intake of $800 \mathrm{mg}$ and $1600 \mathrm{mg}$ tablets have been analyzed and the excreted amount of these compounds was calculated from the total amount of urine. As a result, the 10H2DA and 10HDAA were not detected in any fraction $\left(<5 \mathrm{ng} \mathrm{mL}^{-1}\right.$ : data not shown), but 2-DA, SA, and 3-HSA were detected in each urine fraction up to $12 \mathrm{~h}$ after ingestion of ETRJ tablets. The total amount of 2-DA, SA, and 3-HSA was $5879.70 \pm 2103.78$ $\mu \mathrm{g}, 814.68 \pm 351.40 \mu \mathrm{g}$, and $1077.47 \pm 512.60 \mu \mathrm{g}$ after intake of $800 \mathrm{mg}$ ETRJ tablets and $8665.71 \pm 3687.71 \mu \mathrm{g}, 2191.41 \pm$ $957.83 \mu \mathrm{g}$, and $1647.79 \pm 1062.10 \mu \mathrm{g}$ after intake of $1600 \mathrm{mg}$ ETRJ tablets, respectively (Fig. 4). 

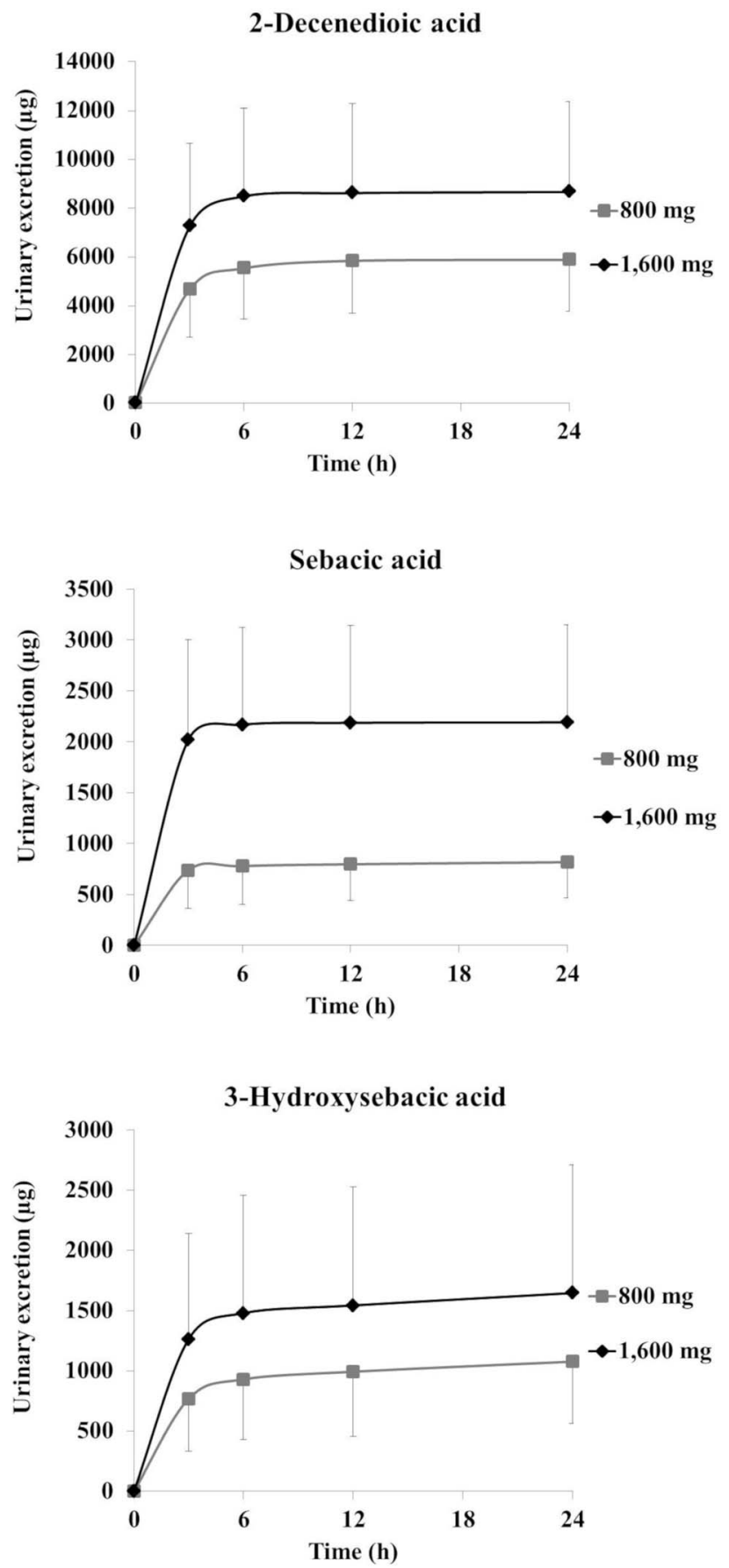

Fig. 4 Total amount of urine excreted 2-decenedioic acid, sebacic acid, and 3-hydroxysebacic acid in human subjects (mean \pm SDs; $n=12$ ) $0-24 \mathrm{~h}$ after ingestion of $800 \mathrm{mg}$ or $1600 \mathrm{mg}$ ETRJ tablets.

\section{Discussion}

This study revealed that fatty acids in RJ were metabolized to dicarboxylic acids, absorbed into the circulation and then excreted in the urine. We also demonstrated that fatty acids in ETRJ were absorbed to a greater extent than those of RJ.

Metabolome analysis of plasma after RJ capsules consumption revealed dicarboxylic acids but not hydroxy fatty acids ingested from RJ, such as: 10H2DA, 10HDAA, and 3,10-dihydroxydecanoic acid. This result indicates that the terminal hydroxyl group of each fatty acid should be oxidized by phase I metabolism in the liver and converted into dicarboxylic acids. Generally, fatty acids undergo two types of oxidation. The most common pathway is stepwise catabolism via $\beta$-oxidation and formation of acetyl-CoA. The second type of oxidation are $\omega$ oxidation where the terminal methyl and methylene position of fatty acids is undergoing conversion to carboxyl. In case of lauric acid, a medium chain fatty acid, it was reported that around $20 \%$ of laurate is estimated to undergo $\omega$-oxidation. ${ }^{23}$ In contrast, almost all of the absorbed 10H2DA and 10HDAA could have undergone $\omega$-oxidation because there were no significant increases in plasma concentration values of 10H2DA and 10HDAA before and after RJ intake, suggesting that RJ fatty acids could be efficiently $\omega$-oxidized compared with other MCFAs, maybe because of the terminal hydroxyl groups.

In this study, we also demonstrated that the gastrointestinal absorption of RJ fatty acids increases with protease treatment of RJ. In the previous study, medium and long chain fatty acids are reported to bind to albumin. ${ }^{24}$ In the same way RJ fatty acids might bind to RJ proteins, leading to a low concentration of free fatty acids in the gut and a low passive absorption rate.

Quantitative analysis of RJ plasma metabolites using ETRJ tablets revealed that the AUC values of 2-DA, SA, and 3-HSA were dose dependent (Fig. 3). The total amounts of urinary 2DA, SA, and 3-HSA over $24 \mathrm{~h}$ after RJ intake were also dose dependent and completely excreted within $12 \mathrm{~h}$ (Fig. 4), showing that these metabolites could be useful biomarkers of RJ absorption. The pharmacokinetic parameters of RJ fatty acid metabolites were compared between men and women, and the $C_{\max }$ values of women were 1.2- to 1.5 -fold higher than those in men (Fig. 3 and Table 5). However, considering that men had 1.2-fold higher weights (Table 1), there would be no gender difference in absorption rate. Further study, such as setting the dose per body weight to reduce the individual differences will be needed to conclude the gender difference.

SA is known to have pharmacological activities, estrogenic effect via estrogen receptor $\beta,{ }^{25}$ TRPA1 and TRPV1 activation, ${ }^{26}$ increases GLUT-4 expression in L6 myoblast, ${ }^{27}$ and anti-inflammatory effect by inhibition of inducing TNF- $\alpha .^{28}$ The activity of 2-DA has not been reported so far but might be expected to have similar activities like those described above. In addition, because it has been reported that isotope labeled SA administered intravenously to mice was eliminated about $25 \%$ as $\mathrm{CO}_{2}$ in expired air, ${ }^{29}$ it is possible that dicarboxylic acids to be further metabolized to other components such as ketone body. Similarly, other metabolites generated from those dicarboxylic acids could be responsible for the mechanisms of RJ pharmacological activities. Although further investigation will be needed to determine which components exert positive pharmacological effects of RJ in clinical trials, RJ fatty acid metabolites may be responsible for the pharmacological functions of RJ. 
Table 5 Pharmacokinetic parameters of plasma fatty acid metabolites of female and male volunteers after consumption of $800 \mathrm{mg}$ and $1600 \mathrm{mg}$ ETRJ tablets ${ }^{a}$

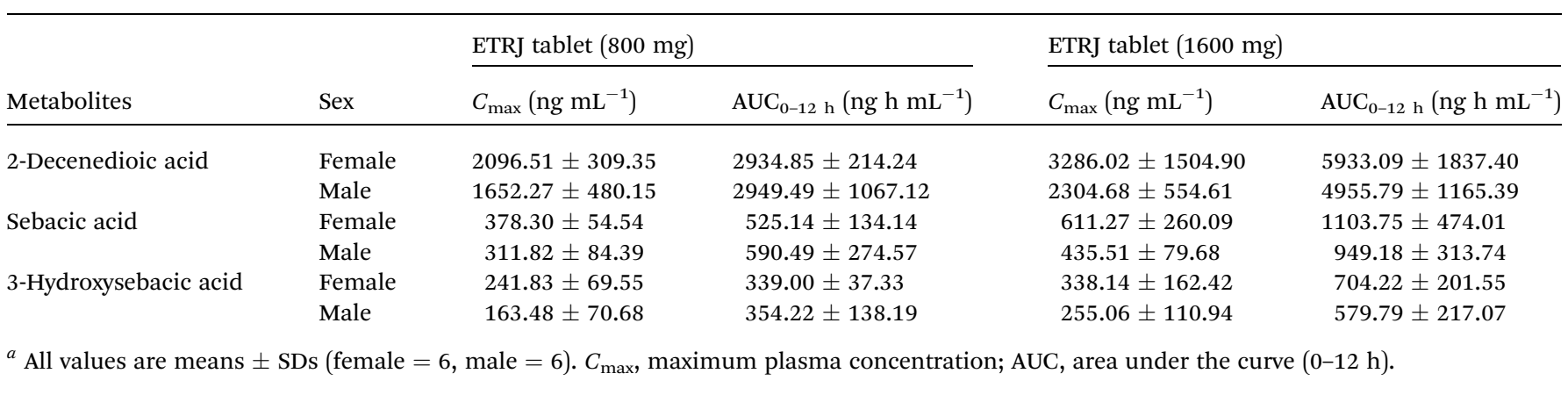

\section{Conclusions}

This study has been established that dicarboxylic acids and their hydroxyl derivatives are absorbed into the circulation and then excreted in the urine. We also found that the fatty acids absorption increased by protease treatment of RJ. Thus, the protease treated RJ will be a promising material not only safe but also effective. Our study clarifies for the first time the absorption, metabolism, and pharmacokinetics of fatty acids derived from RJ in human. This work provides the potential of $\mathrm{RJ}$ for therapeutic application from the aspects of pharmacokinetics and metabolites and supports studies aimed at clarifying the identity of bioactive RJ constituents and their biological effect.

\section{Contribution of authors}

The authors' responsibilities were as follows - MY: designed the study, conducted most of the experimental work, and contributed to the drafting of the manuscript; HT: contributed to the experimental work and helped revise the manuscript; AY, TT and $\mathrm{KH}$ : supervised the study design and the experimental work, and contributed to the drafting of the manuscript and its revision.

\section{Conflict of interest}

There are no conflicts of interest to declare.

\section{Abbreviations}

AUC
$C_{\max }$
2-DA
ETRJ
10H2DA
10HDAA
3-HSA
MCFAs
RJ
SA

\author{
Area under the concentration \\ Maximum plasma concentration \\ 2-Decenedioic acid \\ Enzyme treated royal jelly \\ 10-Hydroxy-2-decenoic acid \\ 10-Hydroxydecanoic acid \\ 3-Hydroxysebacic acid \\ Medium chain fatty acids \\ Royal jelly \\ Sebacic acid
}

$\begin{array}{ll}t_{1 / 2} & \text { The elimination half-life } \\ T_{\max } & \text { The time to reach } C_{\max }\end{array}$

\section{Acknowledgements}

We thank Dr Ana-Maria Oprea for helping us to proofread English the manuscript and $\mathrm{Mr}$ Masashi Nakamichi for preparing test food.

\section{References}

1 C. I. Pavel, L. A. Marghitas, O. Bobis, D. S. Dezmirean, A. Sapcaliu, I. Radoi and M. N. Madas, Biological activities of royal jelly-review, Anim. Sci. Biotech., 2011, 44, 108-118.

2 G. F. Townsend, J. F. Morgan, S. Tolnai, B. Hazlett, H. J. Morton and R. W. Shuel, Studies on the in vitro antitumor activity of fatty acids. I. 10-hydroxy-2-decenoic acid from royal jelly, Cancer Res., 1960, 20, 503-510.

3 Y. Nakajima, K. Tsuruma, M. Shimazawa, S. Mishima and H. Hara, Comparison of bee products based on assays of antioxidant capacities, BMC Complementary Altern. Med., 2009, 9, 11258.

4 K. Kohno, I. Okamoto, O. Sano, N. Arai, K. Iwaki, M. Ikeda and M. Kurimoto, Royal jelly inhibits the production of proinflammatory cytokines by activated macrophages, Biosci., Biotechnol., Biochem., 2004, 68, 138-145.

5 J. M. Tseng, J. R. Huang, H. C. Huang, J. T. Tzen, W. M. Chou and C. C. Peng, Facilitative production of an antimicrobial peptide royalisin and its antibody via an artificial oil-body system, Biotechnol. Prog., 2011, 1, 153-161.

6 H. M. Park, M. H. Cho, Y. Cho and S. Y. Kim, Royal jelly increases collagen production in rat skin after ovariectomy, J. Med. Food, 2012, 6, 568-575.

$7 \mathrm{~N}$. Yamada and H. Yoshimura, Effect of royal jelly on chills in Japanese young women, J. Jpn. Soc. Nutr. Food Sci., 2010, 63, 271-278.

8 T. Tatefuji, T. Asama, S. Doi, T. Sugano and K. Hashimoto, Efficacy of royal jelly supplement on neck muscle strain (Japanese: Katakori) in healthy adult women: a randomized double-blind, placebo-controlled trial, East. J. Med., 2010, 26, 55-64. 
9 Y. Dake, Y. Okuno, K. Okihara, K. Hashimoto and T. Enomoto, Efficacy of royal jelly supplement on tinnitus treatment : A randomized open controlled trial, Pharmacometrics, 2009, 75, 109-116.

10 T. Asama, H. Matsuzaki, S. Fukushima, T. Tatefuji, K. Hashimoto and T. Takeda, Royal jelly supplementation improves menopausal symptoms such as backache, low back pain and anxiety in postmenopausal Japanese women, J. Evidence-Based Complementary Altern. Med., 2018, 2018, 4868412.

11 G. Meng, H. Wang, Y. Pei, Y. Li, H. Wu, Y. Song, Q. Guo, S. Fukushima, T. Tatefuji, J. Wang, H. Du, Q. Su, W. Zhang, S. Shen, X. Wang, R. Dong, P. Han, T. Okazaki, R. Nagatomi, J. Wang, G. Huang, Z. Sun, K. Song and K. Niu, Effects of protease-treated royal jelly on muscle strength in elderly nursing home residents: A randomized, double-blind, placebo-controlled, dose-response study, Sci. Rep., 2017, 7, 11416.

12 H. Guo, A. Saiga, M. Sato, I. Miyazawa, M. Shibata, Y. Takahata and F. Morimatsu, Royal jelly supplementation improves lipoprotein metabolism in humans, J. Nutr. Sci. Vitaminol., 2007, 53, 345-348.

13 T. Sugiyama, K. Takahashi and H. Mori, Royal jelly acid, 10hydroxy-trans-2-decenoic acid, as a modulator of the innate immune responses, Endocr., Metab. Immune Disord.: Drug Targets, 2012, 12, 368-376.

14 V. A. Isidorov, S. Bakier and I. Grzech, Gas chromatographicmass spectrometric investigation of volatile and extractable compounds of crude royal jelly, J. Chromatogr. B: Anal. Technol. Biomed. Life Sci., 2012, 885-886, 109-116.

15 G. F. Townsend, W. H. Brown, E. E. Felauer and B. Hazlett, Studies on the in vitro antitumor activity of fatty acids. IV. The esters of acids closely related to 10-hydroxy-2-decenoic acid from royal jelly against transplantable mouse leukemia, Can. J. Biochem. Physiol., 1961, 39, 1765-1770.

16 T. Maeda, H. Kuroda and K. Motoyoshi, Effects of royal jelly and 10-hydroxy decenoic acid on the sebaceous glands of hamster ear, Nihon Hifuka Gakkai Zassi, 1988, 98, 469-475.

17 S. Koya-Miyata, I. Okamoto, S. Ushio, K. Iwaki, M. Ikeda and M. Kurimoto, Identification of a collagen productionpromoting factor from an extract of royal jelly and its possible mechanism, Biosci. Biotechol. Biochem., 2004, 68, 767-773.
18 M. S. Blum, A. F. Novak and S. Taber III, 10-Hydroxy-delta 2decenoic acid, an antibiotic found in royal jelly, Science, 1959, 130, 452-453.

19 S. Ito, Y. Nitta, H. Fukumitsu, H. Soumiya, K. Ikeno, T. Nakamura and S. Furukawa, Antidepressant-like activity of 10-hydroxy-trans-2-decenoic acid, a unique unsaturated fatty acid of royal jelly, in stress-inducible depression-like mouse model, J. Evidence-Based Complementary Altern. Med., 2012, 2012, 139140.

20 J. C. Newman and E. Verdin, Ketone bodies as signaling metabolites, Trends Endocrinol. Metab., 2014, 1, 42-52.

21 T. Moriyama, M. Yanagihara, E. Yano, G. Kimura, M. Seishima, H. Tani, T. Kanno, T. Nakamura-Hirota, K. Hashimoto, T. Tatefuji, T. Ogawa and Y. Kawamura, Hypoallergenicity and immunological characterization of enzyme-treated royal jelly from Apis mellifera, Biosci., Biotechnol., Biochem., 2013, 77, 789-795.

22 E. Melliou and I. Chinou, Chemistry and bioactivity of royal jelly from Greece, J. Agric. Food Chem., 2005, 53, 8987-8992.

23 W. Kam, K. Kumaran and B. R. Landau, Contribution of $\omega$ oxidation to fatty acid oxidation by liver of rat and monkey, J. Lipid Res., 1978, 19, 591-600.

24 A. A. Spector, Fatty acid binding to plasma albumin, J. Lipid Res., 1975, 16, 165-179.

25 P. Moutsatsou, Z. Papoutsi, E. Kassi, N. Heldring, C. Zhao, A. Tsiapara, E. Melliou, G. P. Chrousos, I. Chinou, A. Karshikoff, L. Nilsson and K. Dahlman-Wright, Fatty acids derived from royal jelly are modulators of estrogen receptor functions, PLoS One, 2010, 5, e15594.

26 Y. Terada, M. Narukawa and T. Watanabe, Specific hydroxy fatty acids in royal jelly activate TRPA1, J. Agric. Food Chem., 2011, 59, 2627-2635.

27 A. Iaconelli, A. Gastaldelli, C. Chiellini, D. Gniuli, A. Favuzzi, C. Binnert, K. Mace and G. Mingrone, Effect of oral sebacic acid on postprandial glycemia, insulinemia, and glucose rate of appearance in type 2 diabetes, Diabetes Care, 2010, 33, 2327-2332.

28 Y. F. Chen, K. Wang, Y. Z. Zhang, Y. F. Zheng and F. L. Hu, In vitro anti-inflammatory effects of three fatty acids from royal jelly, Mediators Inflammation, 2016, 2016, 3583684.

29 P. A. Tataranni, G. Mingrone, A. De Gaetano, C. Raguso and A. V. Greco, Tracer study of metabolism and tissue distribution of sebacic acid in rats, Ann. Nutr. Metab., 1992, 36, 296-303. 